\title{
Examinations for overseas-trained dentists in Australia and the UK: formative and summative feedback
}

\author{
Youngha Song ${ }^{1}$
}

\section{Key points}

Helps understand examinations for overseas-trained dentists in Australia and the UK.
Provides formative and summative feedback on the examinations from a participant and practitioner's perspective.
Proposes three suggestions to better evaluate the competency of overseas-trained dentists in Australia and the UK.

\begin{abstract}
Examinations for overseas-trained dentists are enforced to qualify for registration to perform dental practices in some countries. Feedback on the examinations in Australia and the UK is presented as formative and summative evaluations from a participant and practitioner's perspective. The formats of both examinations are analysed with the foci of the composition, implementation and standard-setting/standardisation in practical tests. The structures of the examinations are formulated in a different manner, resulting in different pass rates. Some administrative errors and loopholes are identified in the implementation. The issue of reliability is raised for the acceptability of the practical examination. Among components of the examinations, establishing the relationship and communicating with patients is more valued to practitioners trained overseas, along with medical emergency protocols for patients' safety. To better evaluate the competency of overseas-trained dentists in Australia and the UK, three suggestions are proposed. Firstly, the examination governing body should ask for and refer to feedback from actively practising dentists passing through the qualification process. Next, the examinations should redirect the target of competency from dental manikin-based dexterity to a more comprehensive evaluation. Finally, the equivalent level of qualifying competency for the examinations described in official publications may need to be revisited.
\end{abstract}

\section{Introduction}

Dental qualifications are generally recognised within only the home country or certain foreign areas under the valid accreditation of dental education. Thus overseas-trained dentists (OTDs) - qualified in other than the destination country and in which their primary dental education was not performed - need to either pass specific examinations or take further training programmes if seeking registration for dental practice. A few countries enforce the requirement of successful examination results on OTD candidates; these include Australia, ${ }^{1}$ the United Kingdom, ${ }^{2}$ Canada, ${ }^{3}$ and South Korea. ${ }^{4}$ Each country has its own dental accreditation authority governing the examinations: the Australian

${ }^{1}$ Australian Research Centre for Population Oral Health, University of Adelaide, South Australia, Australia.

Correspondence to: Youngha Song

Email: youngha.song@adelaide.edu.au

Accepted 25 February 2019

DOI:10.1038/s41415-019-0371-y
Dental Council (ADC) exam in Australia; the Overseas Registration Examination (ORE) by the General Dental Council in the UK (commissioned to the examination suppliers); ${ }^{5}$ the National Dental Examining Board of Canada (NDEB) Equivalency Process in Canada; and the Korea Health Personnel Licensing Examination Institute in South Korea.

The international migration of OTDs has become a global issue, beyond specific agreements or partnerships in a local region. ${ }^{6}$ For some high-income countries, the number of qualified OTDs occupies a significant portion of the oral health workforce. For example, one out of four practising dentists in Australia is an OTD, ${ }^{7}$ and 1,300 dentists had qualified through ORE between 2007 and 2016 in the UK. ${ }^{5}$ In particular, the issue of 'Brexit' may lead to huge ramifications for the examination in the UK - currently primary dental qualifications for practice within the European Economic Area are mutually and automatically recognised across countries. Not only for a country's policy on the dental care system, but for individual candidates, the examinations for OTD are 'high-stakes' attempts at the cost of a considerable amount of resources. ${ }^{5}$ Given its importance on both sides, however, there are very few study findings, ${ }^{7}$ except for the official publications from the examination governing entity. If any, they are either 'floating' tips from interest groups on the internet or unproven advice from private preparation courses and institutions for the examinations.

This opinion piece is to present personal arguments on the examinations for OTD in Australia and the UK. They are based on anecdotal evidence and 'snapshot' analyses. However, as a former participant in both the ADC examination and the ORE, I happen to occupy a vantage point to reflect on the pitfalls and propose suggestions for improvement. Having been educated up to undergraduate level and practised dentistry for six years in South Korea, I passed through the ADC exam at the second attempt in 2014. Since carrying out dental practices in Australia, I have attempted the ORE twice in 2014 and 
Table 1 Comparison in formats of the ORE, pre-/post-revision of the ADC exam and the NDEB Assessment of Clinical Skills (as of 2019 January, otherwise specified)

\begin{tabular}{|c|c|c|c|c|}
\hline & ORE & ADC exam (2018 Jul ) & ADC exam (2014 Jun 2018 Mar) & NDEB \\
\hline $\begin{array}{l}\text { Application and } \\
\text { requirements }\end{array}$ & $\begin{array}{l}\text { Application form with: } \\
\text { Clinical experience (1,600 hrs); } \\
\text { Evidence of English language }\end{array}$ & No change & $\begin{array}{l}\text { Initial assessment: } \\
\text { Evidence of English language (when } \\
\text { applying for registration after exam pass) }\end{array}$ & NDEB Equivalency Process \\
\hline Knowledge test & $\begin{array}{l}\text { Part } 1 \text { (for } 2 \text { days): } \\
\text { Two papers of 3-hour time } \\
\text { limit each; } \\
\text { Multiple short answer } \\
\text { questions }\end{array}$ & No change & $\begin{array}{l}\text { Written examination (for } 2 \text { days): } \\
\text { Four papers of } 2 \text {-hour time limit each; } \\
80 \text { multiple choice questions each }\end{array}$ & $\begin{array}{l}\text { Assessment of fundamental } \\
\text { knowledge: } \\
\text { Two books of } 150 \text { multiple choice } \\
\text { questions for } 3 \text { hrs each. } \\
\text { Assessment of clinical judgement: } \\
\text { Two books of } 60 \text { single/multiple } \\
\text { choice questions for total } 5 \text { hrs. }\end{array}$ \\
\hline $\begin{array}{l}\text { Practical and } \\
\text { clinical skills test }\end{array}$ & $\begin{array}{l}\text { Part } 2 \text { (for } 3 \text { days) with four } \\
\text { components: } \\
\text { An operative test on a dental } \\
\text { manikin (DM); } \\
\text { An objective structured clinical } \\
\text { examination (OSCE); } \\
\text { A diagnostic and treatment } \\
\text { planning exercise (DTP); } \\
\text { A practical examination in } \\
\text { medical emergencies (ME) }\end{array}$ & $\begin{array}{l}\text { Practical examinations (for } \\
2 \text { days) with } 16 \text { tasks: } \\
\text { Clinical skills day (OSCE } 10 \\
\text { stations); } \\
2 \text { x clinical information } \\
\text { gathering; } \\
2 \times \text { diagnosis and management } \\
\text { planning; } \\
6 \text { x clinical treatment and } \\
\text { evaluation. } \\
\text { Technical skills day (dental } \\
\text { models } 6 \text { tasks): } \\
3 \times \text { restorative-based; } \\
3 \times \text { preparation-based. }\end{array}$ & $\begin{array}{l}\text { Practical examinations* (for } 2 \text { days) with } \\
13 \text { tasks out of } 14 \text { : } \\
\text { Restoration; } \\
\text { class II composite resin, } \\
\text { class IV composite resin, } \\
\text { class II amalgam. } \\
\text { Preparation; } \\
\text { class III composite resin, } \\
\text { class II amalgam, } \\
\text { full gold crown, } \\
\text { metal-ceramic crown. } \\
\text { Endodontic access. } \\
\text { Fabrication of a provisional crown. } \\
\text { Applying a rubber dam. } \\
\text { Record keeping. } \\
\text { Infection control. } \\
\text { Clinical communication. } \\
\text { Taking radiographs in a manikin. }\end{array}$ & $\begin{array}{l}\text { Assessment of clinical skills* (for } \\
2 \text { days) with } 12 \text { tasks: } \\
\text { Restoration; } \\
\text { class II composite resin, } \\
\text { class IV composite resin, } \\
\text { class II amalgam. } \\
\text { Preparation; } \\
\text { class III composite resin,. } \\
\text { class II amalgam, } \\
\text { full metal crown, } \\
\text { metal-ceramic crown. } \\
\text { Endodontic access. } \\
\text { Fabrication of a provisional crown. } \\
\text { Dental dam application. } \\
\text { Record keeping. } \\
\text { Infection control and safety. }\end{array}$ \\
\hline
\end{tabular}

2018, for only personal achievements, which left unsuccessful results but valuable findings for this paper. To provide more context, my less than optimal proficiency in the English language and unfamiliarity with dental education in western countries can help to justify my arguments on what and how the competency is evaluated in the examinations for dentists trained overseas. Many colleague candidates contributed to the arguments for this paper by sharing and discussing their 'unofficial' ideas, which have a lot in common with my own.

\section{Formative feedback}

For every candidate, the format of the examination and how to pass it are clearly of the utmost importance. This section is feedback on how candidates are assessed by the examination in the actual test venue. In spite of considerable differences in dental education and service systems among countries, the overall format of the examinations appears mostly consistent. The stages comprise a series of an initial qualification assessment, dental knowledge test, and clinical skills test in a chronological order (Table 1). They are seemingly posited on the consensus about the evaluation of learning outcomes in dentistry (for example, both the ADC exam and the ORE are based on Miller's framework of 'Knows', 'Knows how', 'Shows how', 'Does'). ${ }^{8}$ As can be seen in Table 1, the first stage (initial document assessment/verification) and second stage (written test) of the qualification process are less different from each other than the clinical tests are. In practice, little dissonance and few complaints about the written examinations are observed among candidates, which is supported by relatively high pass rates. For the ADC exam, $35 \%$ in written and $22 \%$ in clinical tests during 2014-2018; for the ORE, 63\% and $39 \%$, respectively, over the same period (calculated on the arithmetic mean of published pass rates). That is presumably interpreted by the nature of multiple choice questions from established item banks and fair marking standards for a pass result. Therefore, this paper will be mainly about practical/clinical tests in formative and summative feedback as well as suggestions. Three foci in this section are the composition, implementation and standard setting/standardisation of the examination.

\section{ADC exam}

For the past five years, there have been two major revisions in the ADC exam, mostly on the format of the practical examination. The first revision was in 2014, with the adoption of an analogous composition to that of the NDEB Assessment of Clinical Skills (ACS) executed in Canada. Out of the 14 tasks in the ADC practical exam, 12 tasks were almost identical to those in the NDEB, including the marking criteria. Despite the addition of a 'clinical communication' task, the ADC practical exam was mostly bound in a dental manikin (DM)based assessment, which contrasts with a more comprehensive evaluation in the NDEB. In Canada, the certification process requires all licensure candidates, including graduates of accredited dental programmes, sit further written examination and objective-structured clinical examination (OSCE), even after the pass result in the NDEB ACS for overseas-trained dentists. To align the examination format with the learning outcomes stated in the Professional competencies of the newly qualified dentist, ${ }^{9}$ the ADC practical exam has been revised into two methods of assessment - OSCE and DM tasks - from June 2018 onwards. The inclusion of the 
OSCE format in the practical examination can be conducive to the assessment of candidates' overall competencies for the fitness of dental practice with reference to the statement.

In the former revision, the implementation of the ADC practical exam produced few issues except for administrative errors in the beginning of the then new format (for example, poor quality of the tooth for the endodontic access or lack of manikin stability for taking radiographs). The time limit and scope of tasks were not restrictive for candidates' performance in the practical examination. By the nature of the DM-based test, however, some 'tricks and tips' for better marks 'worked' in the task, in spite of their counterproductive practice for patients (for example, the bulk filling of composite resin for aesthetics and convenience rather than incrementally filling to reduce polymerisation shrinkage, and the restorative contour with hypo-occlusion in the manikin as practically not being marked). For the latter revision, feedback from the implementation should be acquired to minimise unnecessary errors and loopholes in the evaluation.

If candidates' performance, the difficulty of tasks, and examiners' marking standards are the same, the result of the examination is expected to be the same. However, although almost identical tasks with the same rubric and marking criteria were given as aforementioned, the pass rate of the ADC practical exam and the NDEB ACS were much different from 2014 to 2018 (22\% and 38\%, respectively). That can either be because, in the ADC exam, two different tasks out of 14 items were decisively difficult, the candidates' abilities were significantly poor, or examiners' standard setting was harsher than in the NDEB. It can be fair for each destination country to have different levels of competency for dental practice, in accordance with different circumstances in their oral health care system. Nevertheless, it should be noted that a reliability issue in the practical examination may occur even under very similar conditions if not calibrated properly, such as being prone to measurement biases in epidemiological studies.

\section{Overseas registration examination}

The composition of the ORE part two is more consistent and comprehensive than that of the ADC practical exam. The ORE part two consists of four components which can evaluate candidates' competency - learning outcomes aligned with the Preparing for practice document, ${ }^{10}$ - in a disparate and thorough format. The extensive scope and diverse modality of the examination compared with only DM-based tests creates a heavy burden but does not necessarily lead to a poorer result (the pass rate of 39\% in the ORE part two and $22 \%$ in the ADC practical exam from 2014 to 2018). Rather, it can be more beneficial to the actual dental practices, not for the sake of the examination, to be described in sections of summative feedback and suggestions.

The ORE part two is implemented in a pragmatic manner for each format of components from a candidate's perspective. A diagnostic and treatment planning exercise (DTP) and DM allow a sufficient amount of time; 54 minutes and three hours, respectively. 13 minutes for a medical emergency (ME) exercise may seem very tightly time-limited, but the nature of prompt reaction to emergencies can justify the implementation. However, some stations in OSCE require candidates to demonstrate the tasks based on only rote memory during a five-minute time limit (for example, handwriting a referral letter for uncommon oral diseases with reference to an exhaustive list of marking criteria). For those tasks, the format of OSCE in the NDEB-extended match-type questions can be considered to assess candidates' clinical skills in a limited time.

One of the candidates' major concerns about the examination is the reliability of the test result-standardisation. As most practical examinations have inherent limitations of discerning pass or fail results, candidates on the 'borderline' around the 'cut-score' may raise a reliability issue rather than those with an explicit level of satisfactory/unsatisfactory performance. ${ }^{5}$ Although the borderline regression has been adopted in both the ADC exam $^{11}$ and the ORE, ${ }^{5}$ many candidates still wonder what constitutes the fail result in practice. Even those with the pass result are sometimes confused about the marks given. It is not unusual, from candidates' accounts, that a critical fault in DM tasks, described clearly on marking criteria, resulted in the pass and vice versa. Among those in disagreement on the criteria are experienced dental specialists trained in their home country with relevant sub-disciplines such as operative dentistry and prosthodontics. In addition, for DM in the ORE part two, only three exercises are tested (two major and one minor) which may cause an issue of internal consistency reliability compared with the NDEB and the ADC exam (six to ten tasks). Therefore, establishing more robust and transparent marking standards with greater reliability is urged for better acceptability of the practical examination to the candidates.

\section{Summative feedback}

In contrast to the previous formative feedback, this section is about how the examinations are assessed from a practitioner's perspective in dental practice settings. Luckily, I have passed through the ADC exam and been carrying out dental practices in Australia for over three years. Also, I can assume that my feedback on the ORE, based on the experience in Australia, remains valid to the UK. That is because of the similarity in dental practice, based on the facts that UK-educated dentists are eligible to apply for the registration, being exempt from the ADC exam, and dentists from the UK have been practising without many clinical difficulties in Australia. My findings follow the format of four components in the ORE for its better-balanced classification on the evaluation of competencies than a mostly DM-based assessment of the ADC exam in 2014.

As an OTD practising in Australia, what I value the most among the components in the examination is DTP. Despite six years of clinical practices in my home country, I find the most different and difficult competency being how to establish the relationship, and communicate with culturally and linguistically diverse patients in Australia. The communication skills and management of oral complaints learned through DTP preparation are salient to adapt to the local context of the dental care system. The component of ME provides important learning opportunities as well. It does not have a lot to do with daily dental practices but equips me with the protocol on medical emergencies in the dental setting. In particular, the protocol carries more weight as the need for extra care is common in dental practice, due to an increasingly ageing population with polypharmacy as well as patients with chronic diseases.

On the other hand, the assessment of DM tasks has little merit to dental practice in Australia. It is not because clinical skills on DM are of less value to patients but because they are much more common as a universally compatible competency from pre-existing experiences in a home country. That is to say, DM-based tests are effective for learning outcomes to the students in dental education but not as 
important as the evaluation of trans-cultural/ system adaptation for experienced dentists. Regarding the OSCE component, it may be less helpful to the evaluation depending on how and what to test. Currently, a few OSCE stations are repeatedly tested from item banks, and candidates sometimes need to carry out certain tasks without a thought for the marking criteria within an insufficient time. In order to align the assessment and cover a wide sample of learning outcomes, ${ }^{5}$ OSCE stations should be diversified and marked on the basis of wellstructured/organised formats for evaluation.

\section{Suggestions}

Inferred from the findings in formative and summative evaluations, I would suggest three points to both the ADC exam and the ORE: seeking feedback from practising dentists registered through the examinations; redirection away from the DM-based assessment; and amendment of expected competencies for the examinations. Most feedback on the examinations is from the candidates who have been through the process. Thus, they mainly tend to be about the difficulty of scoring passable marks in the exam, rather than the improvement of the evaluation for the competencies conducive to the actual dental practice. Furthermore, those comments may lack validity if affected by either acquiescence bias (for example, 'yes-saying') or personal circumstance-based complaints (for example, tasks which they felt were unfair or unexpected but justifiable from the statement of learning outcomes). The examination governing body needs to both seek, and refer to, feedback from actively practising dentists passing through the qualification process. As in the saying 'danger past, god forgotten', once the examination ends up with the pass result, those successfully registered are not inclined to look back and analyse the exam. However, the feedback from more objective and practical viewpoints would become a salutary source for the improvement of the examination, different from those with the expertise in the evaluation of education.

Next, the examinations should redirect the target of competency from DM-centred dexterity to a more comprehensive evaluation. For the ADC practical exam, the format of postrevision appears to be a better composition, with the adoption of OSCE stations rather than the previously DM dominance, but still draws heavily upon intraoral skills. The ORE seems to be structured with four balanced components in the format. In practice, however, the pass rate of DM is critical to the overall result compared with the other components (49\% for DM vs 77\% DTP, 74\% ME and 63\% OSCE from 2014 to 2018). The diversification and recalibration of the current dependency upon $\mathrm{DM}$ are triangulated by the rationale already raised; commonality across different dental care systems, harsher marking results than the criteria indicated, and concerns about the reliability of marking DM tasks. If the aim of the examinations for OTD is to protect the public, ${ }^{5}$ every aspect of dental practice should be equally evaluated, not sending a wrong signal to the candidates that DM is the crucial determinant for the qualification. In this regard, DM tasks need to be tested on the adjusted weighting and standard setting comparable to those of the other components.

Finally, the equivalent level of qualifying competency for the examinations described in official handbooks and guidance may need to be revisited. The competencies expected of candidates are presented as 'a recently qualified Australian dental practitioner at the point of graduation from an ADC-accredited dental program'11 or 'the standard of a "just passed" UK BDS graduate.' ${ }^{2}$ It is reasonable to set a reference point for the evaluation of learning outcomes in education. However, the standards can mislead candidates to two comparability problems. By introducing the same competency level, a concern could arise as to whether ADC-accredited dental graduates and UK BDS graduates can qualify through the ADC exam and the ORE, respectively, as past candidates for the examinations did (that is, direct comparison). That is in line with the principle of equivalence or non-inferiority trial that the comparator's efficacy should be demonstrated first in order to vindicate the result. Another is for the indirect comparison that different levels of competency for different countries are laid on the same comparable scale by adopting the same standards. For example, a candidate qualifying in the ORE may fail in the ADC exam for the fair reason of different competency levels required for different countries. However, as noted above, currently UK BDS graduates are exempt from the ADC exam for registration in Australia. If ORE qualifying candidates are recognised with the same competency of 'just passed' UK BDS graduates, it can be contradictory as a double standard between OTD and UK-educated dentists for registration in Australia. Therefore, I suggest for the qualifying competency that candidates are expected to 'perform competent translation of general dental knowledge and clinical skills into the proper context of the [country's] dental care system'

\section{Acknowledgements}

The author is supported by Australian Government Research Training Program Scholarship. The author deeply acknowledges critical comments on the manuscript by Pf David Brennan, Australian Research Centre for Population Oral Health, The University of Adelaide.

\section{References}

1. Australian Dental Council. Dentist assessments. Available at https://www.adc.org.au/PractitionerAssessments/Dentist-Assessments accessed May 2019).

2. General Dental Council. Overseas registration exam. Available at https://www.gdc-uk.org/professionals/ore (accessed May 2019).

3. The National Dental Examining Board of Canada. Examination/Assessment Resources. Available at https://ndeb-bned.ca/en/resources/ ExaminationAssessmentResources (accessed May 2019)

4. Korea Health Personnel Licensing Examination Institute. Preliminary examination for DLE. Available at http://www.kuksiwon.or.kr/EngHome/context. aspx?page=sub_2_1\&sub=25 accessed May 2019).

5. Bissell V, Chamberlain S, Davenport E, Dawson L, Jenkins S, Murphy R. The Overseas Registration Examination of the General Dental Council. Br Dent J 2016; 221: 257-261.

6. Balasubramanian M, Brennan D S, Spencer A J, Short S D. The international migration of dentists: directions for research and policy. Community Dent Oral Epidemiol 2016; 44: 301-312.

7. Balasubramanian M, Brennan D S, Spencer A J, Watkins K, Short S D. Overseas-qualified dentists' experiences and perceptions of the Australian Dental Council assessment and examination process: the importance of support structures. Aust Health Rev 2014; 38: 412-419.

8. Miller $\mathrm{G}$ E. The assessment of clinical skills/competence/ performance. Acad Med 1990; 65 (Spec Iss): S63-S67.

9. Australian Dental Council. Professional competencies of the newly qualified dentist. 2016. Available from https:// www.adc.org.au/sites/default/files/Media_Libraries/ PDF/Accreditation/Professional\%20Competencies $\% 20$ of $\% 20$ the $\% 20$ Newly $\% 20$ Qualified\%20Dentist_rebrand. pdf (accessed May 2019).

10. General Dental Council. Preparing for Practice. 2015 Available at https://www.gdc-uk.org/professionals/ students-and-trainees/learning-outcomes (accessed May 2019).

11. Australian Dental Council. ADC assessment process. 2018. Available at https://www.adc.org.au/ ResourceAndPublications/AccreditationFormsDocuments (accessed May 2019). 